\title{
Pseudotumour cerebri syndrome due to cryptococcal meningitis
}

\author{
Phillip D Cremer, Ian H Johnston, G Michael Halmagyi
}

\begin{abstract}
Three cases are reported of the pseudotumour cerebri syndrome-that is, intracranial hypertension without mass lesion or enlarged ventricles, due to cryptococcal meningitis. In these patients the papilloedema was successfully treated with optic nerve sheath decompression, and the intracranial hypertension with lumboperitoneal CSF shunting. These cases support the concept that pseudotumour cerebri is a syndrome of intracranial hypertension that can be due to any disorder producing obstruction of the CSF pathways at the level of the arachnoid villi. This concept is important because it directs therapy to normalise the intracranial pressure and preserve vision.
\end{abstract}

(F Neurol Neurosurg Psychiatry 1997;62:96-98)

Keywords: pseudotumour cerebri; intracranial hypertension; cryptococcus; meningitis

\section{Case reports}

Pseudotumour cerebri is a syndrome of intracranial hypertension, without a mass lesion or enlarged ventricles. In fat young women it is usually idiopathic. Several conditions known to interfere with CSF absorption pathways can produce the pseudotumour syndrome. ${ }^{1}$ It is important to distinguish whether intracranial hypertension is due to pseudotumour syndrome or hydrocephalus, as the usual treatments of pseudotumour syndrome-optic nerve sheath decompression or lumboperitoneal shunting - are contraindicated in hydrocephalus. We report three patients with cryptococcal meningitis who developed severe intracranial hypertension due not to hydrocephalus but to pseudotumour syndrome.

Neurosciences, Roya

Prince Alfred

Hospital, Sydney,

Australia

P D Cremer

I $\mathrm{H}$ Johnston

G M Halmagyi

Correspondence to:

Dr Phillip D Cremer,

Department of Neurology,

Royal Prince Alfred Hospita

Camperdown NSW 2050,

Sydney, Australia.

Received 5 January 1996

and in revised form

7 May 1996

Accepted 13 May 1996

MRI showed meningeal enhancement with gadolinium contrast (fig $1 \mathrm{~A}$ and $\mathrm{B}$ ) but normal sized ventricles. Lumbar puncture disclosed a CSF pressure $>35 \mathrm{~mm} \mathrm{Hg}, 56$ lymphocytes $/ \mathrm{mm}^{3}$, and a protein concentration of $1.38 \mathrm{~g} / 1$ (normal $<0.45 \mathrm{~g} / \mathrm{l}$ ). The venous sinuses were patent on cerebral arteriography. Cryptococci were present on indian ink preparation of the CSF, and the serum cryptococcal antigen titre was $1: 1024$. She was treated with intravenous amphotericin B and 5-flucytosine. After one week of treatment she experienced sudden bilateral visual loss down to the perception of light in each eye, and a partial left abducens nerve palsy. Both optic nerve sheaths were then decompressed.

Within 48 hours of the operation vision began to improve in her left eye. However, the decompression failed to control the intracranial hypertension: ventricular CSF pressure studies showed that after infusion of $9 \mathrm{ml}$ saline, the intracranial pressure rose to $47 \mathrm{~mm}$ $\mathrm{Hg}$ (normal $<20 \mathrm{~mm} \mathrm{Hg}$ ). A lumboperitoneal shunt was inserted, and the repeat pressure study was normal. Visual acuity in the left eye eventually improved to $6 / 12$, but remained at counting fingers in the right, and she developed bilateral optic atrophy.

\section{PATIENT 2}

A previously healthy 35 year old man presented with headache and vomiting for one month, and was found to have bilateral advanced papilloedema, enlarged blind spots, and normal visual acuity. Brain CT was normal, with normal sized ventricles. Lumbar puncture disclosed a CSF pressure $>40 \mathrm{~mm}$ $\mathrm{Hg}, 59$ lymphocytes $/ \mathrm{mm}^{3}$, and a protein concentration of $0.63 \mathrm{~g} / 1$. Cryptococci were present on indian ink preparation of the CSF, and the serum cryptococcal antigen titre was 1:512. Despite treatment with amphotericin B and 5flucytosine for 10 weeks the papilloedema persisted and the CSF pressure remained raised at $36 \mathrm{~mm} \mathrm{Hg}$. The CT remained normal throughout. The venous sinuses were patent on cerebral arteriography. An isotopic CSF study showed that the CSF pathways were patent, with no basal block. A lumboperitoneal shunt was inserted, the intracranial hypertension was relieved, and the papilloedema resolved.

\section{PATIENT 3}




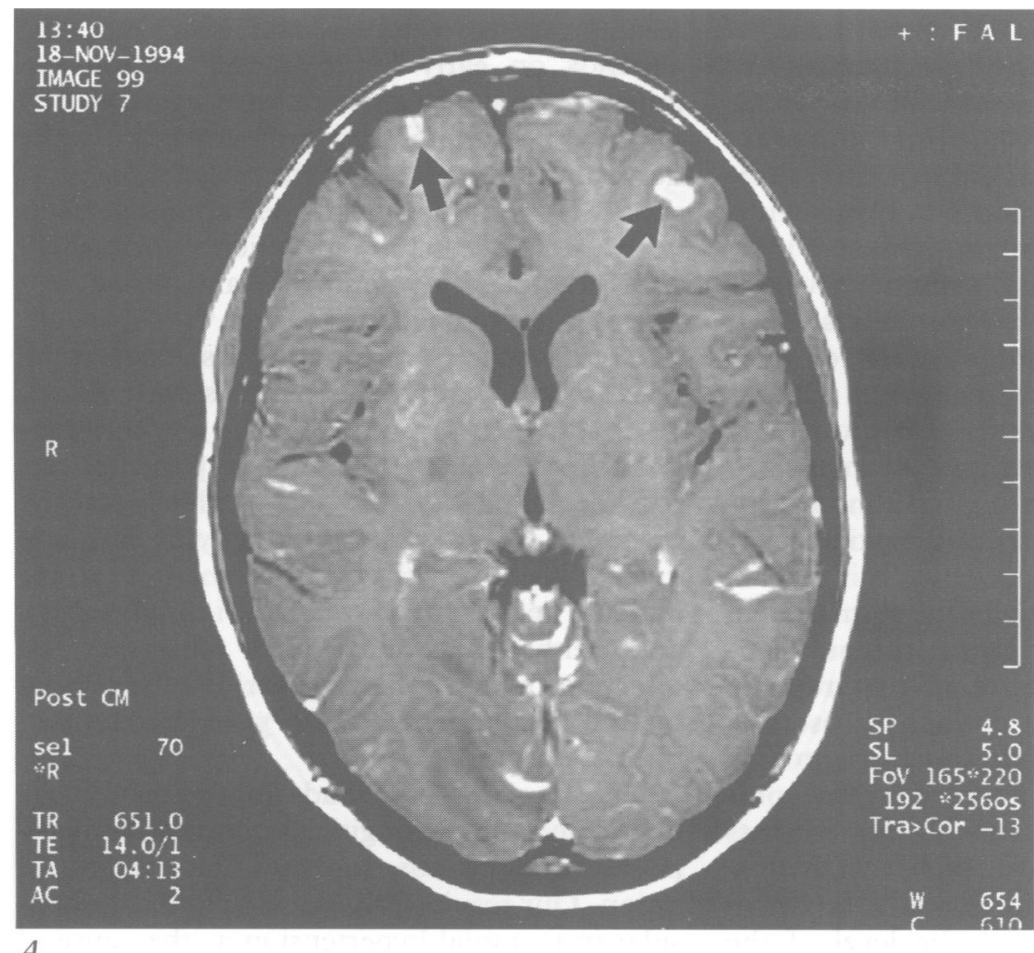

A

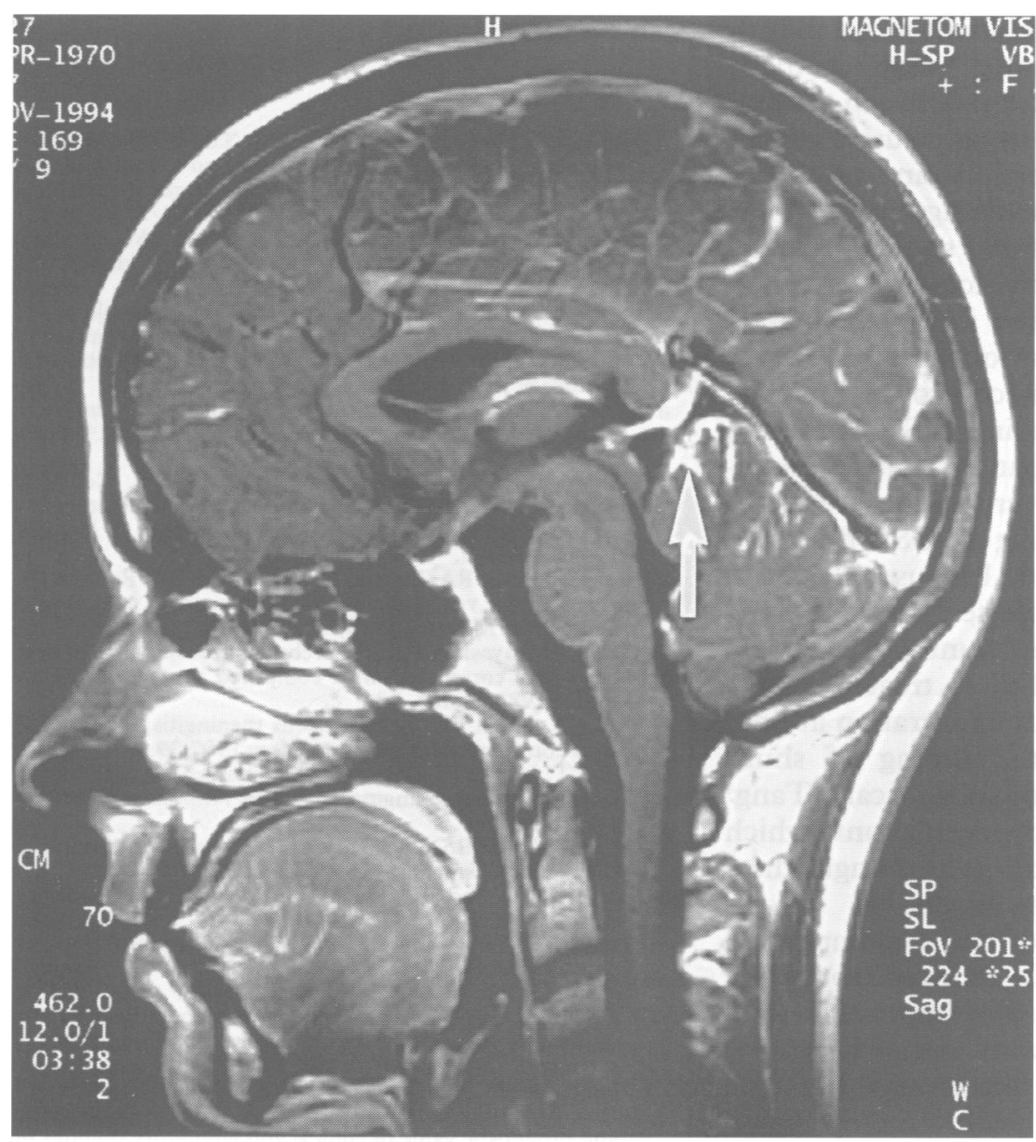

B

Axial (1A) and sagittal (1B) T1 weighted MRI of patient 1 (after gadolinium contrast). There is prominent meningeal enhancement within cortical sulci (black arrows), and around the pons and cerebellum (white arrow). The ventricles are of normal size.

plained of severe headache and vomiting and was found to have bilateral papilloedema, more advanced on the right, with enlarged blind spots and normal visual acuity and colour vision. He also had a partial left abducens nerve palsy. Brain CT was normal, with normal sized ventricles. Brain MRI showed slight swelling of the optic nerve sheaths, but was otherwise normal. Lumbar puncture disclosed a pressure of $35 \mathrm{~mm} \mathrm{Hg}$, no white cells, and a protein concentration of $0.83 \mathrm{~g} / \mathrm{l}$. Numerous Cryptococci were seen on indian ink preparation of the CSF, and the cryptococcal antigen titre exceeded 1:4096 in both serum and CSF. The patient was treated with amphotericin B and acetazolamide. A second lumbar puncture showed persistently raised pressure, so the right optic nerve sheath was decompressed, and the papilloedema began to resolve in both eyes within three days of surgery. However, intracranial pressure remained high and a lumbar CSF infusion study showed a baseline pressure of $19 \mathrm{~mm}$ $\mathrm{Hg}$, with a rise to $>60 \mathrm{~mm} \mathrm{Hg}$ after infusion of normal saline at $30 \mathrm{ml} / \mathrm{h}$.

Over the next four weeks, the papilloedema resolved and eventually the patient developed bilateral optic atrophy. After three months, the visual acuity remained normal and the visual fields remained unchanged. The patient did not have any headaches, and he was generally well. However, after four months he presented with sudden bilateral visual loss due to herpes simplex retinitis, and he then developed bilateral retinal detachment. His visual acuity 12 months after the original cryptococcal meningitis was reduced to the perception of light in the right eye and counting fingers in the left.

\section{Discussion}

Findings on these three patients show conclusively that a syndrome of intracranial hypertension without ventricular enlargement - that is, the pseudotumour syndrome-can occur in cryptococcal meningitis. The mechanism is presumably defective CSF absorption at the level of the arachnoid villi, due to direct inflammation. It is also possible in some cases that raised CSF protein contributes to the failure of CSF absorption; however, the protein concentrations were only slightly raised in our patients.

The strictest definition of pseudotumour cerebri includes only those patients with intracranial hypertension, normal cerebral imaging (in particular no ventriculomegaly or mass lesion), and normal CSF constituents. The typical patient is a fat young woman, with an idiopathic form of the condition. However, there is increasing recognition that this clinical picture could arise from different causes, including cranial venous outflow impairment, drugs (for example, tetracyclines and vitamin A), and chronic idiopathic meningitis. ${ }^{1}$ More recently there have been two studies in which direct cerebral venography and manometry have demonstrated cerebral venous hypertension to be the cause in patients who had previously been labelled "idiopathic". ${ }^{3}$ We argue that the term pseudotumour syndrome ${ }^{1}$ should be used to encompass these disorders for two reasons: firstly, the term implies that the clinical pattern of intracranial hypertension without ventriculomegaly could have various causes. 
Secondly, it emphasises the importance of recognising and treating the raised pressure in its own right, even in the absence of ventricular enlargement, to preserve vision. In the context of cryptococcal meningitis, the fact that blinding intracranial hypertension can occur without ventricular enlargement is not widely recognised. Indeed some groups advocate CSF shunting in cryptococcal meningitis only if the ventricles are enlarged. ${ }^{4}$ Review of published cases of cryptococcal meningitis complicated by intracranial hypertension shows that $30 \%-70 \%$ of these patients have normal sized ventricles, ${ }^{5-8}$ which makes pseudotumour syndrome the rule rather than the exception.

In a patient with papilloedema it is important to determine whether the intracranial hypertension is due to pseudotumour syndrome or to hydrocephalus, to offer appropriate treatment. The treatment options for pseudotumour syndrome include lumboperitoneal CSF shunting and optic nerve sheath decompression, which are not suitable for patients with hydrocephalus. The presence of hydrocephalus implies that there is some degree of obstruction of CSF flow at the level of the basal cisterns or within the ventricular system, requiring direct drainage of the ventricles (for example, with a ventriculoperitoneal shunt). Lumboperitoneal shunting may actually increase the pressure gradient between the cranial cavity and the spinal canal, causing downward herniation of the medulla and cerebellar tonsils through the foramen magnum.

The first case confirms that sudden bilateral blindness can occur in cryptococcal meningitis, ${ }^{5-9}$ due either to raised intracranial pressure $^{9}$ or to direct invasion of the optic nerves by the fungus. ${ }^{10}$ Rigorous control of intracranial hypertension, including CSF shunting, improves vision in most patients with intracranial hypertension due to cryptococcal meningitis. ${ }^{9}$ In theory the timing of CSF diversion could be important, both because placing a foreign body into an infected space is hazardous, and because the shunt might block. These considerations led us to treat patient 1 with an optic nerve sheath fenestration at first. In practice, however, the timing of shunt insertion might not be so critical: Tang ${ }^{4}$ reported his experience of seven shunts which were inserted before starting antifungal therapy; there were no adverse effects.

The optic nerve sheath was decompressed in two of our patients. It reduced optic disc swelling in both patients and improved visual acuity in the patient with reduced acuity, despite failing to reduce intracranial pressure in either. Optic nerve sheath decompression has been used to treat visual loss in idiopathic pseudotumour cerebri, and the outcome depends on early intervention. ${ }^{11}$ Vision has improved despite persistent intracranial hypertension, presumably by protecting the optic nerves from the effects of the raised CSF pressure. ${ }^{11}$ Optic nerve sheath decompression has been used successfully in patients with AIDS with cryptococcal meningitis and visual loss due to intracranial hypertension. ${ }^{12}$

Cryptococcal meningitis is becoming increasingly common in patients with AIDS, yet it still occurs in patients who are immunocompetent $^{13}$ (as in two of our patients). Its presence could reflect the virulence of the organism, or an obscure underlying immune defect. We favour the first explanation for our patients because they were previously healthy, and neither have developed other unusual infections after a follow up period of two years for the first patient and 16 years for the second.

The concept of pseudotumour syndrome due to chronic meningitis has important therapeutic implications for the preservation of vision. Our results emphasise the need to consider intracranial hypertension as the cause of optic disc swelling and visual loss in patients with chronic meningitis, even if the ventricular size is normal.

1 Johnston I, Hawke S, Halmagyi GM, Teo C. The pseudotumour syndrome. Disorders of cerebro-spinal fluid cirtumour syndrome. Disorders of cerebro-spinal fluid circulation causing intracranial hypertension

2 King JO, Mitchell PJ, Thomson KR, Tress BM. Cerebral King JO, Mitchell PJ, Thomson KR, Tress BM. Cerebral
venography and manometry in idiopathic intracranial hypertension. Neurology 1995;45:2224-8.

3 Karahalios DG, Rekate HL, Khayata MH, Apostolides PJ. Elevated intracranial venous pressure as a universal mechanism in pseudotumor cerebri of varying etiologies. Neurology 1996;46:198-202.

4 Tang L-M. Ventriculoperitoneal shunt in cryptococcal meningitis with hydrocephalus. Surg Neurol 1990;33: 314-9.

5 Johnston SRD, Corbett EL, Foster O, Ash S, Cohen J. Raised intracranial pressure and visual complications in AIDS patients with cryptococcal meningitis. $\mathcal{F}$ Infection 1992;24:185-9.

6 Rex JH, Larsen RA, Dismukes WE, Cloud GA, Bennett JE. Catastrophic visual loss due to Cryptococcus neoformans meningitis. Medicine 1993;72:207-24.

7 Daunt N, Jayasinghe LS. Cerebral torulosis: clinical features and correlation with computed tomography. Clin Radiol 1985;36:485-90.

8 Tan CT, Kuan BB. Cryptococcus meningitis, clinical-CT scan considerations. Neuroradiology 1987;29:43-6.

9 Tan CT. Intracranial hypertension causing visual failure in Cryptococcus meningitis. $\Im$ Neurol Neurosurg Psychiatry 1988;51:944-6.

10 Offner S, Baker RS. Visual loss in cryptococcal meningitis. $\mathcal{F}$ Clin Neuro-ophthalmol 1987;7:45-8.

11 Corbett JI, Nerad JA, Tse DT, Anderson RL. Results of optic nerve sheath decompression for pseudo-tumour optic nerve sheath decompression for pseudo-tumour Ophthalmol 1988;106:1391-7.

12 Garrity JA, Herman DC, Imes R, Fries P, Hughes CF, Campbell RJ. Optic nerve sheath decompression for Campbell RJ. Optic nerve sheath decompression for visual loss in patients with acquired immunodeficiency syndrome and cryptococcal meningitis

13 Butler WT, Alling DW, Spickard A, Utz JP. Diagnostic and prognostic value of clinical and laboratory findings in cryptococcal meningitis. N Engl f Med 1964;270:59-67. 